\title{
Ranking the Online Documents Based on Relative Credibility Measures
}

\author{
${ }^{1,2}$ Ahmad Dahlan, ${ }^{1}$ Dwi H. Widyantoro, ${ }^{1}$ Suhono H. Supangkat $\&{ }^{1}$ Benhard Sitohang \\ ${ }^{1}$ School of Electrical Engineering and Informatics - ITB - Indonesia \\ ${ }^{2}$ Department of Electronics and Informatics - TEDC Bandung - Indonesia
}

\begin{abstract}
Information searching is the most popular activity in Internet. Usually the search engine provides the search results ranked by the relevance. However, for a certain purpose that concerns with information credibility, particularly citing information for scientific works, another approach of ranking the search engine results is required. This paper presents a study on developing a new ranking method based on the credibility of information. The method is built up upon two well-known algorithms, PageRank and Citation Analysis. The result of the experiment that used Spearman Rank Correlation Coefficient to compare the proposed rank (generated by the method) with the standard rank (generated manually by a group of experts) showed that the average Spearman $0<r_{S}<$ critical value. It means that the correlation was proven but it was not significant. Hence the proposed rank does not satisfy the standard but the performance could be improved.
\end{abstract}

Keywords: Citation; credibility of online document; document rank; Page Rank.

\section{$1 \quad$ Introduction}

There are at least two problems on the search engines result pages. Firstly, the numbers of irrelevance information that are returned by the search engine $[1,2$, 3]. Secondly there is no guarantee that we can trust the information found $[2,3]$. The first problem is very important to be handled for every information searchers because they need only the relevance information. And the second one should also be managed if they really need credible information, for example searching information for scientific references.

A lot of work has been done to cope with the problem of relevance, such as ranking the search result pages based on their relevance $[1,2,3]$. But the problem of information credibility is still a challenge in information search engine development [4] and remains important areas where research is warranted and timely [5]. This paper proposes a method to rank the information on the search result pages based on their credibility.

Credibility is simply believability [6] or trust in business concept $[7,8]$. Trust is a belief or expectation that the word or promise by the merchant can be relied

Received May $18^{\text {th }}, 2009$. 
upon and the seller will not take an advantage of the consumer's vulnerability. So a credible man is a man who can be trusted, and credible information is information that can be trusted.

According to Fogg \& Tseng [9] having credibility is perceived as having quality because credibility is always perceived from users' perspective. Concerning to on-line information, credibility is how well one can trust the information found on the Internet, as well as on other resources [10].

\section{Criteria of Credibility}

Information or message credibility is generally agreed as a result from an interaction of source characteristics (e.g. expertise, trustworthiness), message characteristics (related to message content, encompassing factors such as plausibility, internal consistency, and quality) and receiver characteristics (e.g. cultural background, previous beliefs) [11]. Regarding to online information, there were three factors to evaluate the credibility of web pages: (1) authority, (2) information content, and (3) web design [3].

Authority is the most important factor in evaluating the credibility of information. Wathen \& Burkell [11] named it source credibility. It shows how well the authority of the source is concerning to its content. People mostly believe information from credible sources. As long as people trust to the "who", it does not matter about the "what". The authority factor can be seen from the credibility of the author, the publishing body, and the link to and from resources [10].

The second factor, the information content, is actually the most difficult factor to be measured. But it is the one that people should care about, because the truth is the truth whoever it comes from. To objectively judge the credibility of the information content, people must have a sufficient understanding about the information itself. But once people have the understanding about it they probably will not search the information from Internet anymore.

In determining the quality of information content, one of the problem is the one's perception depends on a certain situation. For example, if one is searching complete information on a certain topic then detail information will be perceived higher quality than the global one. Familiarization and understanding of the topic also influence users' perception on the quality of information content. This factor can be assessed from its currency, accuracy, objectivity, perspectives, coverage, and its referral to other sources [3]. 
The third factor, web design, is not directly correlated with the quality of information itself, but it is mentioned here because credibility is always perceived from users' perspective. Web design elements such as structure and navigation, visual design, functionality, interactivity, and accessibility affect users' perception on the credibility of the website [3].

\section{Previous Works}

There were several papers dealing with credibility evaluation of online information. Prasad et al [12] proposed three methods to measure the credibility of data in Internet i.e. "Last Update", "Majority Basis", and "Polling" methods. The first method measures newness, a fraction in information content of credibility criteria; the second counts the number of visit and in fact does not measure the credibility of information; and the third is too easy to be manipulated because only the webmaster has access to control the number of votes on the polling.

Huerta \& Ryan [13] found that, by using ELM (Elaboration Likelihood Model) approach, the quality of information content and the reputation of the web owner were the most dominant variables in determining the credibility of online information.

Based on Greer et al. [11], Kirk [14], Smith [15], and Beck [16], Dahlan \& Sitohang [3] induced a set of criteria to evaluate the credibility of online information and proposed a combination of PageRank algorithm of Brin \& Page [17] and Citation count of Garfield [18] to measure it [19]. In fact, PageRank evaluates hyperlinks in web pages that represent a transfer of credibility [6][17]. Credibility transfer can also be performed by citation as typically available in the scientific papers [6]. This paper presents an experiment to rank the search engine result based on the work of [19].

\section{Credibility Measurement}

Considering what Huerta \& Ryan [13] have found, the first two factors of the criteria described above will be utilized further in the proposed method to measure the credibility, i.e., authority $(A)$ and information content (I).

In the real world, because of the amazing capability of human being in analyzing the complexity parameters of information, to define the credibility of information the two factors above can be complementary implemented such as in [19]. It can be understood that a known credible author or a credible publisher normally will not publish bad or low quality information. Moreover, if the credibility of information content is accepted it will not be necessary 
anymore to ask who the author or publisher is. But in the virtual world such as Internet that has very limited condition to represent the cognitive and affective capability of human being, defining the credibility of information requires such a combination of both $\boldsymbol{I}$ and $\boldsymbol{A}$ as proposed in [19]:

$$
C r=I \text { AND } A
$$

However, there is no absolute measure of credibility so it should be measured using a relative scale. Therefore, there is no one can say "the information is credible" but it is suggested to say "this information is more credible than that one". To convey the measurement, this paper implements the method proposed in [19] to combine the PageRank and Citation count and to score the information credibility from 0 to 1 . The higher the score the higher the rank of the document compared to other documents of the same set.

\subsection{PageRank}

PageRank is an algorithm that Google implements to rank the popularity of websites in its index [20]. PageRank value represents how important a page on the web is. PageRank evaluates websites according to a computed value determined by the number of other sites linking to them with respect to the PageRank values of those other sites recursively. It also considers the number of links that come out from the sites.

The underlying theory was that a link from one web page to a web page of another site was in essence a vote for that page [20]. The reason was the webmasters would only link to pages that they thought were interesting and valuable to their viewers. Kleinberg [21] named hub page to the page that refers to other page(s) and authority page to the page that is used as a reference.

Brin \& Page [17] defined the PageRank of page A $\left(\boldsymbol{p}_{\boldsymbol{A}}\right)$ that is being pointed by other pages as :

$$
p_{A}=(1-d)+d\left(p_{w 1} / N_{w 1}+\ldots+p_{w n} / N_{w n}\right)
$$

w1-wn : pages that link to page A

$N \quad: \quad$ the number of links that come out from the page(s)

$\boldsymbol{d} \quad$ : damping factor that can be set between 0-1.

It can be seen that high quality hub page shares high PageRank value to the authority page, and the share will be smaller if the number of outbound links from the hub page increases. 
However, the link - the most important part of the PageRank - in fact does not concern to the content of the document nor the author of the page, but it concerns only to the URL address where the document is stored. So, as a measure of information credibility, PageRank value can be assumed to represent the authority of the publishing body or the server in Internet where the information is stored [10][19].

\subsection{Citation Analysis}

Citation analysis is an important tool used to trace scholarly research, measure impact, and justify tenure and funding decisions [22]. It counts how many times a paper is cited by other paper(s). Citation count provides researchers and administrators with a reliable and efficient indicator for assessing the research performance of authors, projects, programs, institutions, and countries, and the relative impact and quality of their work [22][23].

The use of citation count for evaluating research is based on the assumption that citations are a way of giving credit to and recognizing the value, quality, and significance of an author's work [23][24]. At present, three large databases of bibliographical information are available on the Internet: Web of Science (WOS), Scopus, and Google Scholar [25]. Since citations are mostly related to the content of the references, as a measure of information credibility, citation count can be assumed to represent the credibility of information content [19].

\subsection{Formulation of Credibility}

Credibility score can be formulated by combining PageRank score and citation count [19]. Although they both are derived from referencing by others but each of them represents a unique factor of credibility: PageRank score $(p)$ represents the factor of authority, and the Citation count $(c)$ represents the factor of information content. So according to Equation 1 the credibility of information in Internet can be expressed as in (3).

$$
\begin{array}{ll}
c r_{k} & =\alpha^{*} p_{k}+(1-\alpha)^{*} c_{k} \\
c r_{k} & : \text { credibility score of information on page } \mathrm{k} \\
p_{k} & : \text { PageRank value of the server where page } \mathrm{k} \text { located } \\
c_{k} & \text { : Citation count of a paper on page } \mathrm{k} \\
\alpha & \text { : the weight of PageRank }
\end{array}
$$

\subsection{Experiment}

This experiment aims to evaluate the performance of the method in measuring the credibility of online documents. Since credibility is a relative measure, the 
credibility score ( $\boldsymbol{c r}$ ) of a document should be compared to other documents of the same area of interest or topic. The scores were converted to rankings to form what we call the proposed rank. To evaluate the performance of the method, the proposed ranks were compared to standard ranks, which were the ranks based on the credibility of the documents produced by a number of scientists/experts in their respected fields after reading and reviewing the documents manually.

\subsubsection{The Documents}

The experiment were carried out on a superset of scientific documents that we call the golden standard. It was a composed of 15 sets or topics of scientific documents that were randomly selected from Internet. Every set consisted of 9 to 10 documents and each set were reviewed by one scientist.

The investigation was carried out in a way similar to $k$-fold cross validation with $k=5$. This way each set would be observed 5 times. For each turn, the documents were grouped into 2 subsets: the training subset and the test subset. The training subsets were used to determine the optimum value of $\alpha$ as in Equation 3, and the test subsets were used to measure the performance of the method. The performance measurement requires at least 4 documents for testing, and therefore, 5 to 6 documents would be used for the training session. Figure 1 shows the scheme of the trial for a set of 10 documents.

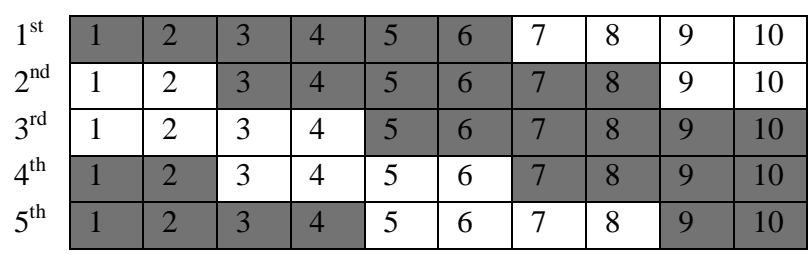

Figure 1 The scheme of the $1^{\text {st }}$ to $5^{\text {th }}$ trial for a set of 10 documents; training subsets, $\square$ test subsets.

\subsubsection{Performance Measure}

This study correlated the proposed rank with the standard rank. The correlation of both ranks was analyzed using Spearman Rank Correlation.

The Spearman's rank correlation coefficient (Spearman $\boldsymbol{\rho}$ or $\boldsymbol{r}_{S}$ ) is a nonparametric measurement correlation. It is used to determine the relation existing between two sets of data [26]. As a nonparametric correlation measurement, it can also be used with nominal or ordinal data. In principle, $\rho$ or $\boldsymbol{r}_{S}$ is simply a special case of the Pearson's product-moment coefficient in which two sets of data $X_{i}$ and $Y_{i}$ are converted to rankings $x_{i}$ and $y_{i}$ before calculating 
the coefficient value. The values of $\boldsymbol{r}_{S}$ are between -1 and +1 : -1 shows a perfect negative correlation, +1 means vice versa, and 0 means there is no correlation [26].

If $x_{i}$ is the rank of value $X_{i i}$ and $y_{i}$ is the rank of value $Y_{\text {iq }}$ the Spearman's rank correlation coefficient denoted by $\boldsymbol{r}_{S}$, can be defined as [26]:

$$
r_{S}=1-\frac{6 \sum d^{2}}{n\left(n^{2}-1\right)}
$$

where:

$$
\begin{aligned}
& n=\text { the size of the sample } \\
& d=x_{i i}-y_{i}
\end{aligned}
$$

In the case where several data have exactly the same values, an average rank will be given to these data ${ }^{1}$.

\subsubsection{Experiment Setup}

The experiment obtained the values of PageRank and Citation count from Google servers and Google Scholar respectively. The credibility score is set to range from 0 to 1 . Since $\boldsymbol{p}_{\boldsymbol{k}}$ (look at Equation 3 ) has been set by Google to range from 0 to 10 , so it should be normalized to range from 0 to 1 . The value of $\boldsymbol{c}_{k}$ should also be normalized and set to range from 0 to 1 . There are two options in normalizing the value of $\boldsymbol{c}_{\boldsymbol{k}}$ : (1) the cumulative option, and (2) the average option.

1) For the cumulative option:

$$
\begin{gathered}
C_{k}=\frac{c_{k}}{\max \left(c_{i}\right)} \\
\text { where } \boldsymbol{i}=1 . . n
\end{gathered}
$$

2) For the average option:

$$
a v\left(c_{k}\right)=\frac{c_{k}}{\left(\text { year }_{\text {now }}-\text { year }_{\text {pub }}\right)}
$$

\footnotetext{
${ }^{1}$ If there are many average ranks, a correction to the formula should be implemented. The correction is not applied here because we work with only small number of samples.
} 


$$
C_{k}=\frac{a v\left(c_{k}\right)}{\max \left(a v\left(c_{i .}\right)\right)}
$$

where $\boldsymbol{i}=1 . . n$

with:

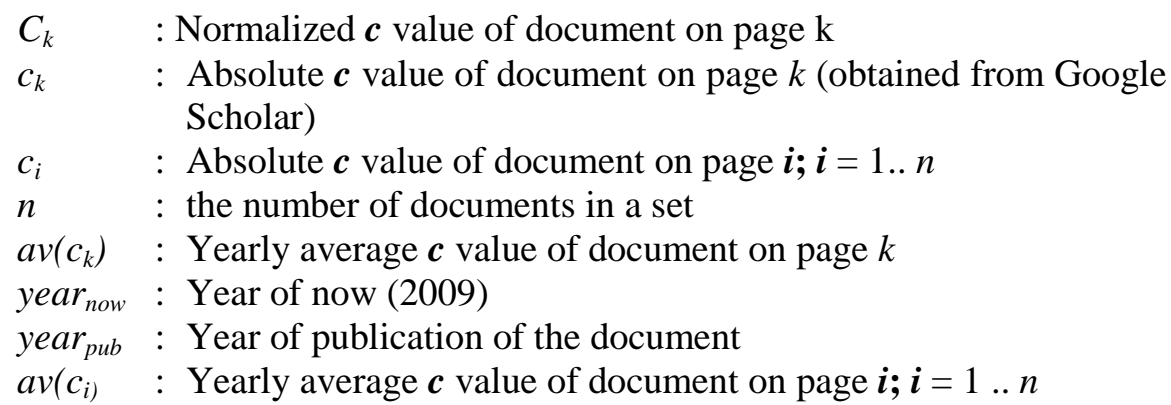

( $\boldsymbol{\alpha}$ is set from 0 to 1 ; with $\boldsymbol{\alpha}=0$ : $\boldsymbol{c r}$ depends only to $\boldsymbol{c}, \boldsymbol{\alpha}=0.5$ : equal weight of $\boldsymbol{p}$ and $\boldsymbol{c}$, and $\boldsymbol{\alpha}=1: \boldsymbol{c r}$ depends only to $\boldsymbol{p}$ ).

\subsubsection{Preliminary Experiment}

The purpose of this step was to obtain the optimum value(s) of $\boldsymbol{\alpha}$ to be used for the experiment with the test subsets. The observation was carried out using the training subsets for all of the fifteen topics. During this session, the credibility score of every document was calculated according to Equation 3 where $\boldsymbol{\alpha}$ was varied from 0 to 1 with increment of 0.1 for both cumulative option and average option. The documents were then ranked according to $\boldsymbol{c r}$ values. The ranks of 5 to 6 documents for each topic were then compared to the standard ranks of the same documents to determine the $\boldsymbol{r}_{S}$ value. Hence, for one topic in each trial there would be 11 records of $\boldsymbol{r}_{\boldsymbol{S}}$ value.

After five trials, the average values of $\boldsymbol{r}_{S}$ were then calculated for each value of $\boldsymbol{\alpha}$. The optimum value of $\boldsymbol{\alpha}$ is the value where $\boldsymbol{r}_{\boldsymbol{S}}$ value is maximum.

This experiment yielded two types of $\boldsymbol{\alpha}$, i.e.: specific $\boldsymbol{\alpha}$ for each topic (written as $\boldsymbol{\alpha}_{s}$ ) and global $\boldsymbol{\alpha}$ for all topics (written as $\boldsymbol{\alpha}_{g}$ ). If there were two or more equal maximum $\boldsymbol{r}_{\boldsymbol{S}}$ values, $\boldsymbol{\alpha}$ value was determined by averaging all of $\boldsymbol{\alpha}$ indicated by the maximum $\boldsymbol{r}_{\boldsymbol{S}}$ values. There would be only one specific $\boldsymbol{\alpha}$ value for each topic, and one global $\alpha$ value for all topics. These values of $\alpha$ would be used to determine the $\boldsymbol{r}_{S}$ values in the test set.

The list of specific $\boldsymbol{\alpha}$ values ( $\boldsymbol{\alpha}_{s}$ ) for both cumulative option and average option, as a result from this preliminary experiment, are shown in Table 1. The 
experiment also found the global $\boldsymbol{\alpha}$ values $\left(\boldsymbol{\alpha}_{g}\right)$ of both cumulative option and average option coincidentally $=0.1$.

Table 1 The list of specific $\alpha$ values $\left(\alpha_{s}\right)$.

\begin{tabular}{|c|c|c|}
\hline Topics & Cumulative Option & Average Option \\
\hline 1. Artificial Intelligence & 1.00 & 1.00 \\
\hline 2. Bayesian Network & 0.10 & 0.90 \\
\hline 3. Biomedical Informatics & 1.00 & 1.00 \\
\hline 4. Cognitive Systems & 0.30 & 0.20 \\
\hline 5. Computer Networks & 0.00 & 0.30 \\
\hline 6. Data Mining & 0.05 & 0.10 \\
\hline 7. Expert Systems & 0.05 & 0.30 \\
\hline 8. Guidance and Counselling & 0.00 & 0.10 \\
\hline 9. Human Machine Interface & 0.00 & 0.00 \\
\hline 10. Information Retrieval & 0.00 & 0.50 \\
\hline 11. Information Search Engine & 1.00 & 1.00 \\
\hline 12. Information Systems & 0.00 & 0.05 \\
\hline 13. Mobile Applications & 1.00 & 0.00 \\
\hline 14. Next Generation Networks & 0.70 & 0.00 \\
\hline 15. Recommender Systems & 0.90 & 0.60 \\
\hline Average & 0.407 & 0.403 \\
\hline
\end{tabular}

\subsubsection{Results}

By applying the same procedure as implemented to the training subsets, the $\boldsymbol{c r}$ scores of all documents were calculated using the defined $\boldsymbol{\alpha}_{s}$ and $\boldsymbol{\alpha}_{g}$ found at the preliminary experiment.

Table 2 shows the average $\boldsymbol{r}_{\boldsymbol{S}}$ values from the trials for both the cumulative option and the average option. The last row shows the average $\boldsymbol{r}_{S}$ values of all topics. For the cumulative option: column 3 shows the $\boldsymbol{r}_{S}$ values with $\alpha=\alpha_{s}$, columns 4 shows the $\boldsymbol{r}_{S}$ values with $\boldsymbol{\alpha}=\boldsymbol{\alpha}_{g}$, and columns 2 and 5 show the comparison of $\boldsymbol{r}_{S}$ values with $\boldsymbol{\alpha}=\mathbf{0}$ and $\boldsymbol{\alpha}=\mathbf{1}$. Columns 6 to 9 show the same figures for the average option.

Table 2 The $r_{S}$ values of the test subset with the defined $\alpha$.

\begin{tabular}{rcccccccc}
\hline \multirow{2}{*}{\begin{tabular}{c} 
Topics \\
\cline { 2 - 10 }
\end{tabular}} & \multicolumn{3}{c}{ Cumulative Option } & \multicolumn{5}{c}{ Average Option } \\
\hline 1 & 2 & 3 & 4 & 5 & 6 & 7 & 8 & 9 \\
\hline 1. & -0.200 & 0.428 & -0.240 & 0.428 & 0.353 & 0.445 & 0.393 & 0.445 \\
2. & 0.848 & $\underline{\mathbf{0 . 8 4 8}}$ & 0.848 & 0.526 & 0.727 & $\underline{\mathbf{0 . 7 2 7}}$ & 0.687 & 0.494
\end{tabular}




\begin{tabular}{rcccccccc}
\hline \multirow{2}{*}{$\begin{array}{r}\text { Topics } \\
\text { r }\end{array}$} & \multicolumn{4}{c}{ Cumulative Option } & \multicolumn{5}{c}{ Average Option } \\
\cline { 2 - 9 } 1 & $\boldsymbol{\alpha}=\mathbf{0}$ & $\boldsymbol{\alpha}=\boldsymbol{\alpha}_{\boldsymbol{s}}$ & $\boldsymbol{\alpha}=\boldsymbol{\alpha}_{\boldsymbol{g}}$ & $\boldsymbol{\alpha}=\mathbf{1}$ & $\boldsymbol{\alpha}=\mathbf{0}$ & $\boldsymbol{\alpha}=\boldsymbol{\alpha}_{\boldsymbol{s}}$ & $\boldsymbol{\alpha}=\boldsymbol{\alpha}_{\boldsymbol{g}}$ & $\boldsymbol{\alpha}=\mathbf{1}$ \\
\hline 3. & -0.404 & -0.068 & -0.278 & -0.068 & -0.640 & -0.068 & -0.640 & -0.068 \\
4. & 0.440 & $\underline{\mathbf{0 . 7 2 0}}$ & 0.520 & -0.017 & 0.400 & $\underline{\mathbf{0 . 6 0 0}}$ & 0.520 & -0.017 \\
5. & 0.880 & $\underline{\mathbf{0 . 8 8 0}}$ & 0.800 & 0.322 & 0.480 & $\underline{\mathbf{0 . 6 8 0}}$ & 0.560 & 0.322 \\
6. & 0.344 & 0.344 & 0.344 & -0.688 & 0.090 & 0.090 & 0.114 & -0.612 \\
7. & 0.360 & 0.360 & 0.360 & 0.060 & 0.080 & 0.160 & 0.080 & 0.060 \\
8. & 0.474 & 0.474 & 0.394 & -0.481 & 0.720 & $\underline{\mathbf{0 . 6 8 0}}$ & 0.680 & -0.481 \\
9. & 0.110 & 0.110 & 0.200 & -0.108 & 0.200 & 0.200 & 0.120 & -0.108 \\
10. & -0.200 & -0.200 & -0.200 & -0.190 & -0.120 & -0.040 & -0.120 & -0.190 \\
11. & -0.160 & 0.065 & -0.160 & 0.065 & -0.240 & 0.065 & -0.240 & 0.065 \\
12. & 0.000 & 0.000 & -0.080 & -0.345 & 0.255 & 0.255 & 0.255 & -0.351 \\
13. & -0.280 & -0.150 & -0.280 & -0.150 & -0.035 & -0.035 & -0.035 & -0.128 \\
14. & -0.240 & -0.160 & -0.240 & -0.256 & -0.120 & -0.120 & -0.120 & -0.256 \\
15. & 0.400 & $\underline{\mathbf{0 . 8 4 0}}$ & 0.400 & 0.738 & 0.520 & $\underline{\mathbf{0 . 7 6 0}}$ & 0.520 & 0.738 \\
\hline Avrg & $\mathbf{0 . 1 5 8}$ & $\mathbf{0 . 2 9 4}$ & $\mathbf{0 . 1 5 9}$ & -0.026 & $\mathbf{0 . 1 7 8}$ & $\mathbf{0 . 2 9 3}$ & $\mathbf{0 . 1 8 5}$ & $\mathbf{- 0 . 0 2 1}$ \\
\hline I.
\end{tabular}

*) The topics are as on Table 1 .

\subsubsection{Discussion of the Results}

It can be inferred from the records in Table 2 that:

(1) According to the critical table from Zar [27], with $\boldsymbol{n}=4$ and significance level $=0.25$ (one tail test $)^{2}$, the $\boldsymbol{\rho}$ critical value $=0.600$; so all average $\boldsymbol{r}_{S}$ values for both option are lower than the critical value, or $0<r_{S}<$ critical value. It means that eventhough there are correlations between the proposed ranks and the standard ranks but the correlations are not significant.

(2) With $\boldsymbol{\alpha}=\boldsymbol{\alpha}_{s}$, in both options, $\boldsymbol{r}_{S}$ values for all topics are equal or greater than both $\boldsymbol{r}_{S}$ values with $\boldsymbol{\alpha}=\mathbf{0}$ and $\boldsymbol{\alpha}=\mathbf{1}$. It means the yielded values of $\boldsymbol{\alpha}_{s}$ produced maximum credibility scores for every topic.

(3) With $\boldsymbol{\alpha}=\boldsymbol{\alpha}_{g}$, in both options, the average $\boldsymbol{r}_{S}$ values (the last row of column 4 and column 8) are equal or greater than the average $\boldsymbol{r}_{S}$ values with both $\boldsymbol{\alpha}=\boldsymbol{0}$ and $\boldsymbol{\alpha}=1$. It means the yielded values of $\boldsymbol{\alpha}_{g}$ produced maximum average credibility scores of all topics.

\footnotetext{
${ }^{2} \mathrm{n}=4$ was taken because there were 4 documents in all topics for the test subsets, and significance level of 0.25 is the lowest one in the critical table.
} 
(4) The positive and high $\boldsymbol{r}_{S}$ values records, such as in topic number 2, 4, 5, 8 and 15 , show positive correlations. It means the proposed ranks are, in certain points, met to the standard ranks. Since $0\left\langle\boldsymbol{r}_{S}\right\rangle$ critical value, it can be stated statistically that for those topics there are correlations between the proposed ranks and the standard ranks, and the correlations are significant.

(5) The all negative $\boldsymbol{r}_{S}$ values records, such as in topic number 3,10,13, and 14, show negative correlations. It means the proposed ranks are opposed to the standard ranks.

(6) Refer to Table 1 , all values of $\boldsymbol{\alpha}$ are mostly equal or closed to 0 or 1 . It means the score depends extremely on one side: Citation count or PageRank, not both. The data related to those aspects that provided to the reviewers were (in addition to the complete document) the names of the authors and the publishers with their url address respectively. Those figures indicate that the judgments in the standard ranks are mainly one side consideration: judgment by the author(s) or judgment by the publisher. Based on the above proposition, the discussion (4) and (5) can be further elaborated.

The positive $\boldsymbol{r}_{S}$ values records, such as mentioned in discussion (4), imply to indication that there were good relations between the judgment and the aspects values. For standard rank with judgment by the author (such as in topic number 5), the documents' scores are directly proportional to Citation counts, and for standard rank with judgment by the publisher (such as in topic number 15) the documents' scores are directly proportional to PageRank values. For example:

- Topic number 5, with 10 documents, the Citation counts are: 56, 149, $69,73,313,68,706,249,1150$, and 186 respectively. The best rank should be: 10, 6, 8, 7, 3, 9, 2, 4, 1, and 5; and the judgments are: 9, 4, 8, $5,7,10,2,3,1$, and 6 . It can be seen that the average judgments have very small difference with Citation counts.

- Topic number 15, with 10 documents, the PageRank values are: 7, 6, 5, $5,7,6,5,6,6$, and 8 respectively. The best rank should be: $2.5,5.5,9,9$, $2.5,5.5,9,5.5,5.5$, and 1 ; and the standard rank is: $1,2,8,5,3,4,9,7$, 10 , and 6. It can be seen that the average judgments have small difference with PageRank values.

The all negative $\boldsymbol{r}_{S}$ values records, such as mentioned in discussion (5), imply to indication that there were misrelations between the judgment and the aspects values. For standard ranks with judgment by the author (such as 
in topic number 10), the documents' scores are inversely proportional to Citation counts, and for standard ranks with judgment by the publisher (such as in topic number 3) the documents' scores are inversely proportional to PageRank values. For example:

- Topic number 10, with 10 documents, the Citation counts are: 73, 528, $585,278,325,77,201,463,289,1131$ respectively. According to those values, the best rank should be $10,3,2,8,5,9,4,7,6$, and 1 ; but the standard rank is: $9,10,7,5,8,2,6,3,1$, and 4 . It can be seen that the average judgments have big differences with Citation counts.

- Topic number 3, with 10 documents, the PageRank values are: 6, 7, 7, 7, $7,8,3,0,7$, and 7 respectively. According to those values, the best rank should be: $8,4.5,4.5,4.5,4.5,1,9,10,4.5$, and 4.5 ; but the standard rank is: $8,9,6,5,4,10,1,7,2$, and 3 . It can be seen that the average judgments have big differences with PageRank values.

\section{Conclusion}

The experiment has shown that there is a correlation between the proposed rank and the standard rank of the documents in the golden standard, but it is not significant. Eventhough the correlations are very low, but the average option has shown a better performance compared to the cumulative option. The experiments also has revealed that the average values of $\boldsymbol{\alpha}$ of both options, i.e.: the average values of specific $\boldsymbol{\alpha}\left(\boldsymbol{\alpha}_{s}\right)$ and the global $\boldsymbol{\alpha}\left(\boldsymbol{\alpha}_{g}\right)$, are <0.5. It means that the credibility score depends more to the Citation value compares to the PageRank value.

Further works suggested to improve the significance of the rank correlation are:

(1) Improving the quality of the golden standard by extending the number of documents, the number of topics, and the number of scientists with the same field of expertise so each set of documents would be reviewed by more than one reviewer. Increasing the number of documents in each set of topic of experiment will increase the probability to result a correlation coefficient in a greater significance level, and increasing the number of scientists with the same field of expertise will improve the quality of the standard ranks of the documents.

(2) Getting the value of the citation count from more than one source, so the citation values are the average of the citation counts from many sources. 


\section{References}

[1] Janes, J.W. \& Rosenfeld, L.B., Networked Information Retrieval and Organization: Issues and Questions, Journal of The American Society for Information Science, 47(9), pp. 711-715, 1996.

[2] Rieh, S.Y., Judgment of Information Quality and Cognitive Authority in the Web, Journal of the American Society for Information Science and Technology, 53, 145-161, 2002.

[3] Dahlan, A. \& Sitohang, B., Kredibilitas Informasi di Internet, Jurnal Gematika, 8(1), 2006.

[4] Knight, S.A. \& Burn, J., Developing a Framework for Assessing Information Quality on the World Wide Web, Informing Science Journal 8 (5), pp. 159-172, 2005

[5] Flanagin, A.J. \& Metzger, M.J., The Role Of Site Features, User Attributes, And Information Verification Behaviors On The Perceived Credibility Of Web-Based Information, New Media and Society, 9(2), pp. 319-342, SAGE Publications, 2007.

[6] Fogg, B.J., Marshall, J., Kameda, T., Solomon, J., Rangnekar, A., Boyd, J. \& Brown, B., Web Credibility Research: A Method for Online Experiments and Early Study Results, Proceedings of ACM CHI 2001 Conference on Human Factors in Computing Systems, v.2. New York: ACM Press, 2001, http://captology.stanford.edu/pdf/WebCred\%20 Fogg\%20 CHI\%202001\% 20short\%20paper.pdf, (18 April 2005).

[7] Geyskens, I., Steenkamp, J.B.E.M., Scheer, L.K. \& Kumar, N., The Effects of Trust and Interdependence on Relationship Commitment: A transatlantic study, International Journal of Research in Marketing, 13, pp. 303-317, 1996.

[8] Jarvenpaa, S.L. \& Tractinsky, N., Consumer Trust in an Internet Store: A Cross-Cultural Validation, Journal of Computer-Mediated Communication vol. 5 issue 2, 2000, http://www. ascusc.org/jcmc/vol5/ issue2/jarvenpaa.html, (18 April 2005).

[9] Fogg, B.J. \& Tseng H., The Elements of Computer Credibility, Proceedings of ACM CHI 99 Conference on Human Factors in Computing Systems, New York: ACM Press: 80-87, 1999, http://captology.stanford.edu/pdf/p80-fogg.pdf, (18 April 2005).

[10] Greer, T., Holinga, D., Kindel, C., Netznik, M., Brown, J., Hickey, K. \& Pozen, V., An Educators' Guide to Credibility and Web Evaluation, White Papers, Universiy of Illinois / Urbana-Campaign 1999, revised on 1st of July 2002, http://lrs.ed.uiuc.edu/wp/ credibility/index.html, (27 April 2005).

[11] Wathen, C.N. \& Burkel, J., Believe it or not: Factors Influencing Credibility on the Web, Journal of American Society of Information Science and Technology, 53(2), pp. 134-144, 2002. 
[12] Prasad, C., Haripriya, Mohania, M., Gupta, A. \& Kambayashi, Y., Credibility of Data in World Wide Web, Advances in Multimedia and Databases for the New Century (Advanced Database Research and Development Series), Vol. 10:10-18, World Scientific, Dec. 1999.

[13] Huerta, E. \& Ryan, T., The Credibility of Online Information, Ninth Americas Conference on Information System, 2003.

[14] Kirk, E.E., Evaluating Information Found on the Internet, Sheridan Libraries John Hopkins University, 1996, http://www.library.jhu.edu/ researchhelp/general/evaluating/ index.html (18 April 2005).

[15] Smith, A.G., Testing the Surf: Criteria for Evaluating Internet Information Resources, The Public-Access Computer Systems Review 8, no. 3, Victoria University of Wellington, NZ; 1997, http://info.lib.uh.edu/ $\mathrm{pr} / \mathrm{v} 8 / \mathrm{n} 3 / \mathrm{smit} 8 \mathrm{n} 3 . \mathrm{html}$ (18 April 2005).

[16] Beck, S.E., The Good, The Bad \& The Ugly: or, Why It's a Good Idea to Evaluate Web Sources, Reference \& Research Services Department New Mexico State University Library, 2005, http://lib.nmsu.edu/instruction/ evalcrit.html, (6 May 2005).

[17] Brin, S. \& Page, L., The anatomy of a large-scale hypertextual Web search engine, Computer Networks and ISDN Systems, 30(1-7), pp. 107117, 1998.

[18] Garfield, E., Citation Indexes for Science, A New Dimension in Documentation through Association of Ideas, Science 122, 108-111, 1955.

[19] Dahlan, A. \& Sitohang, B., Combining PageRank and Citation Analysis to Measure Information Credibility in Internet, Proceedings of Ninth International Conference on Information Integration and Web-based Applications \& Services (iiWAS2007), December 2007.

[20] Sobek, M., The Implementation of PageRank in the Google Search Engine, eFactory Gmbh \& Co. KG Internet Agentur, 2003, http://pr.efactory.de/e-pagerank-implementation.shtml, (6 May 2005).

[21] Kleinberg, J.M., Authoritative Sources in a Hyperlinked Environment, Proceedings of ACM-SIAM Symposium on Discrete Algorithms, pp. 668-677, January 1998.

[22] Yang, K. \& Meho, L.I., Citation Analysis: A Comparison of Google Scholar, Scopus, and Web of Science, Proceeding of the 69th Annual Meeting of the American Society for Information Science and Technology (ASIST) 43, Austin, 2006.

[23] Bauer, K. \& Bakkalbasi, N., An Examination of Citation Count in a New Scholarly Communication Environment, D-Lib Magazine, 11(9), ISSN 1082-9873, 2005, http://www.dlib.org//dlib/september05/bauer/09bauer. html, (7 May 2007).

[24] Borgman, C.L. \& Furner, J., Scholarly communication and bibliometrics, Annual Review of Information Science and Technology, 36, pp. 3-72, 2002. 
[25] Kosmopoulos, C. \& Pumain, D., Citation, Citation, Citation: Bibliometrics, the Web and the Social Sciences and Humanities, Cybergeo, European Journal of Geography, 411, 2007.

[26] Dodge, Y., The Concise Encyclopedia of Statistics, Springer Reference, Springer, 2008.

[27] Zar, J.H., Critical Values of the Spearman's Ranked Correlation Coefficient $\left(r_{s}\right)$, in Biostatistical Analysis, 26, Ed. 2, Table B.19, PrenticeHall, Englewood Cliffs, NJ., 1984, http://webspace.ship.edu/ pgmarr/Geo441/Tables/Spearman\%20Ranked\%20Correlation\%20Table. pdf (18 March 2009). 